\title{
AC 2009-950: HOW MUCH DO THEY REALLY UNDERSTAND? AN ENTRY-LEVEL TEST ON ELECTRICITY AND ELECTROMAGNETICS
}

\section{Chris Smaill, University of Auckland}

Dr Chris Smaill holds a Ph.D. in engineering education from Curtin University of Technology, Australia, and degrees in physics, mathematics and philosophy from the University of Auckland. For 27 years he taught physics and mathematics at high school level, most recently as Head of Physics at Rangitoto College, New Zealand's largest secondary school. This period also saw him setting and marking national examinations, training high-school teachers, and publishing several physics texts. Since the start of 2002 he has lectured in the Department of Electrical \& Computer Engineering at the University of Auckland

\section{Gerard Rowe, University of Auckland}

Dr Gerard Rowe completed the degrees of BE, ME and PhD at the University of Auckland in 1978, 1980 and 1984 respectively. He joined the Department of Electrical and Computer Engineering at the University of Auckland in 1984 where he is currently a Senior Lecturer. He is a member of the Department's Radio Systems Group and his (disciplinary) research interests lie in the areas of radio systems, electromagnetics and bioelectromagnetics. Over the last 20 years he has taught at all levels and has developed a particular interest in curriculum and course design. He has received numerous teaching awards from his institution. In 2004 he was awarded a (National) Tertiary Teaching Excellence Award in the Sustained Excellence in Teaching category and in 2005 he received the Australasian Association for Engineering Education award for excellence in Engineering Education in the Teaching and Learning category. Dr Rowe is a member of the IET, the IEEE, the Institution of Professional Engineers of New Zealand (IPENZ), ASEE, STLHE and AaeE.

\section{Elizabeth Godfrey, University of Auckland}

Dr Elizabeth Godfrey has just finished a 9 year term as the Associate Dean Undergraduate at the School of Engineering at the University of Auckland after a career that has included university lecturing, teaching and 10 years as an advocate for Women in Science and Engineering. She has been a contributor to Engineering Education conferences, and an advocate for the Scholarship of Teaching and Learning since the early 1990s, and is currently President of the Australasian Association of Engineering Education. 


\title{
How much do they really understand? An entry-level test for electricity and electromagnetics
}

\begin{abstract}
First-year engineering numbers have grown considerably over the last decade, and there has been an even greater increase in student diversity. In order to support these students effectively, and to ensure the courses they take remain appropriate, the academic preparedness of these students must be determined. For these reasons, the lecturers in the year-one engineering course Electrical and Digital Systems introduced in 2007 a short diagnostic test at the start of their course to determine the level of understanding of electricity and electromagnetics possessed by the incoming students. This paper presents and discusses the main student misconceptions revealed by the diagnostic test and subsequent investigations, and notes some compelling matches with international research findings.
\end{abstract}

\section{Introduction}

Globally, industry demands an increasing number of engineering graduates. However, the number of high-school students well prepared for studying engineering at university level is shrinking in many countries. Therefore, if freshman engineering numbers are to grow, much of the growth is likely to come from students with lower achievement levels. In order to support these students effectively, and to ensure the courses they take remain appropriate, the academic preparedness of these students must be determined. However, recent changes to the way New Zealand conducts its national examinations have made this more difficult. For example, in the past all students who chose to study physics were required to study a full physics course. Now, the more modular nature of the national examinations enables students to present for examination only some aspects of the physics curriculum while ignoring others. In particular, students could choose not to present the module on electrical systems for examination. In fact, it is now possible for students to enrol in a high-school physics course that contains no electricity content at all.

ELECTENG 101, Electrical and Digital Systems, is part of a compulsory common program for all freshman engineering students at the University of Auckland. Typically 600 students enrol in this course each year, but only about one quarter of them subsequently pursue a degree in electrical and computer engineering. Clearly, many of the students enrolled in ELECTENG 101 may have no particular interest in matters of an electrical nature, and may even have chosen not to study this aspect of physics at high school.

For the reasons outlined above, the lecturers in ELECTENG 101 judged it necessary to introduce a short diagnostic test at the start of their course. Their intention in doing this was to determine the level of understanding of electricity and electromagnetics possessed by the incoming students, and consequently to modify where necessary the course content and its delivery in order to provide the best learning opportunities for the students. It was considered that the test results would identify those students for whom remedial interventions were desirable, and would also indicate the extent and nature of those remedial interventions.

The test was first administered in 2007, and the lecturers were greatly surprised by the extent of the student misconceptions in electricity and electromagnetics that it revealed. The test was administered again in 2008, and the results reinforced those obtained the previous year. 
Follow-up interviews were also conducted in order to gain further insights into student thought processes. Some misconceptions were also revisited in the final examination to determine the extent to which the course had addressed such misconceptions.

The development of the diagnostic test was informed by the results of previous research, much of which has been conducted by the physics-education research community (e.g. Maloney et al ${ }^{1}$, Engelhardt and Beichner ${ }^{2}$, Ding et al ${ }^{3}$, and Saglam and Millar ${ }^{4}$ ). This prior research has identified numerous misconceptions students have about the behavior of DC and AC circuits and about electromagnetics. In the case of DC circuits, commonly identified difficulties include the "current consumption misconception, in which current is viewed as a substance that is "consumed" by a device, such as a light bulb or resistor" 5 and the "sequential reasoning" fallacy ${ }^{2,6}$, in which changes in a circuit component are perceived to affect only later components in the overall circuit.

So far well over 1000 students have sat the diagnostic test, and it is now clear that some misconceptions are common and well-established in the areas of electricity and electromagnetics. This paper presents and discusses the main student misconceptions revealed by the diagnostic test and subsequent investigations, and draws parallels with the results of research conducted elsewhere.

\section{The Diagnostic Test, Initial Analysis}

In 2007 and 2008, the diagnostic assessment was administered (without prior warning) on the second day of the ELECTENG 101 course. This 30-minute assessment consisted of 22 questions. The first 20 questions were multiple-choice while the final two questions were free-response. The multiple-choice questions (worth 1 mark each) covered simple circuit theory (involving batteries, switches, light bulbs and resistors), forces exerted on charges and currents in magnetic fields, and electromagnetic induction. The first free-response question addressed simple circuit analysis (worth 3 marks), while the second addressed simple algebraic manipulation (worth 2 marks). In order to facilitate rapid marking so as to provide prompt feedback to the students, these two free-response questions were marked either right (full marks) or wrong (zero marks). The multiple-choice questions were largely taken from, or based on, assessments previously constructed and used by Engelhardt and Beichner ${ }^{2}$, and Saglam and Millar ${ }^{4}$. The two free-response items were constructed by one of the present authors (Smaill).

In 2007 and 2008 respectively a total of 560 and 543 students completed the 22-question test. The test invigilators reported that the 30 minutes allocated to the test appeared to be ample, as a significant number of students appeared to finish with adequate time to spare. The students also appeared to take the test seriously: they spent most of the allocated 30 minutes working on their answers, and wrote an adequate amount in answering the free-response questions. Mark histograms are provided in Figures 1(a) and 1(b).

In 2007 the mean mark was 10.4 out of 25 , and the pass-rate was $30 \%$. The free-response question which tested year-12 circuit analysis was correctly answered by only $11 \%$ of the students, while the free-response question which tested year-11 algebraic manipulation was correctly answered by only $60 \%$ of the students. (Note that year-13 is the final year of highschool education in New Zealand.) 
Results were very similar for the 2008 cohort, which had a slightly lower entry achievement level: the mean mark was 9.9 , while the pass rate was $27 \%$. Interestingly, these differences persisted through to the final exam, where the pass rate was $93 \%$ in 2007 and $90 \%$ in 2008 . The diagnostic test results for both years were clearly very disappointing. They appeared to indicate that most of the class either had not understood or had forgotten much of the basic physics they had covered in high school.

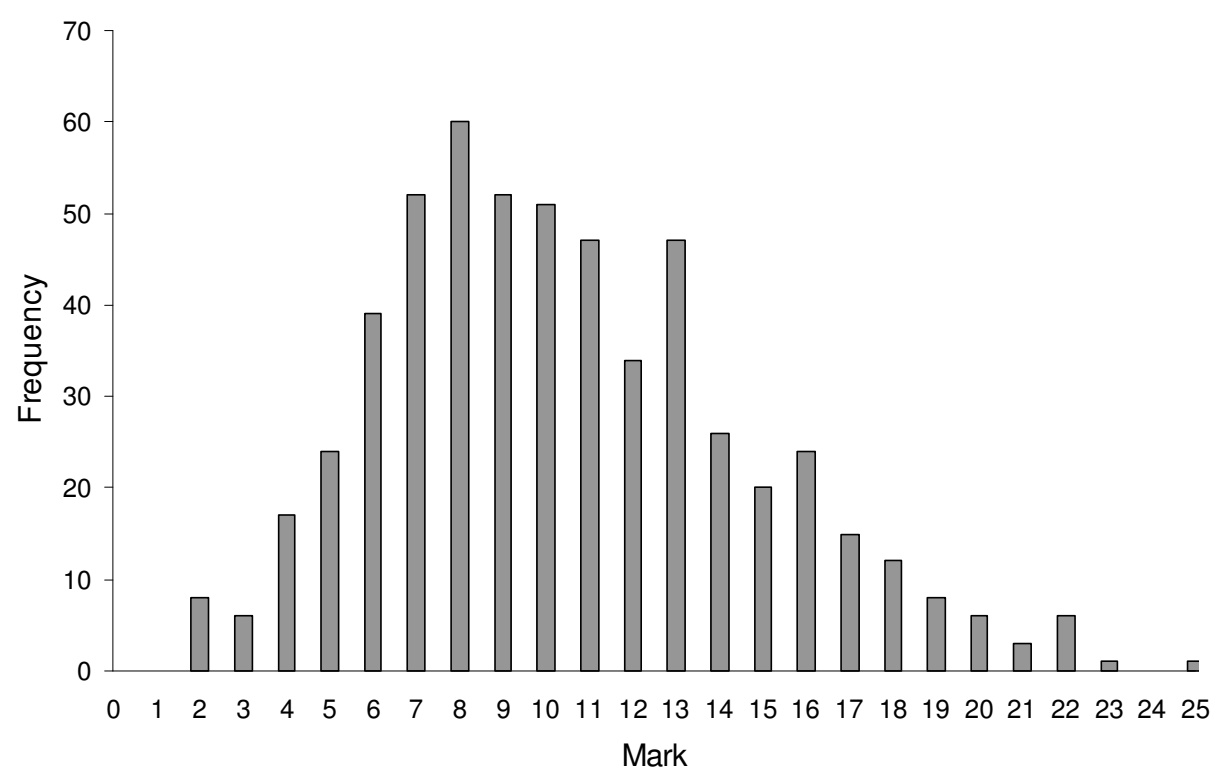

Figure 1(a). Mark histogram for 2007 diagnostic test.

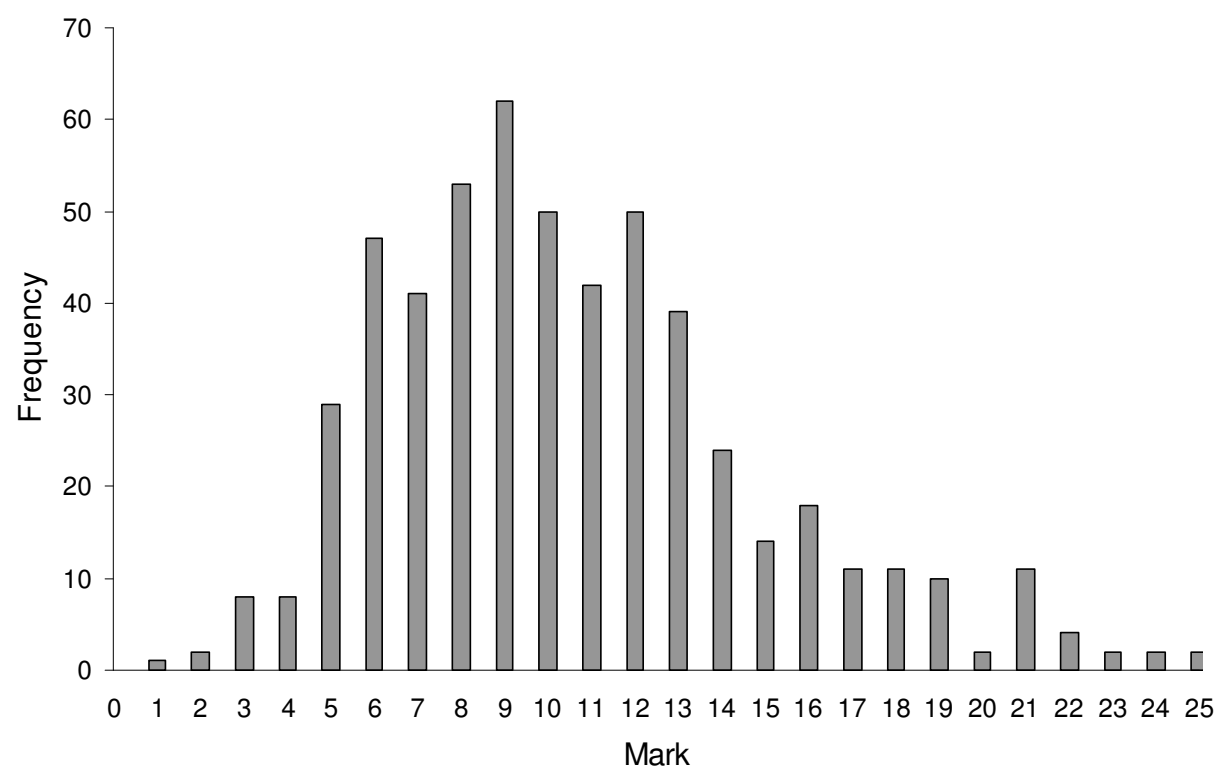

Figure 1(b). Mark histogram for 2008 diagnostic test.

To enable better interpretation of these results, a number of class members were interviewed. In 2007 the interviewer was one of the authors (not known by the students), while in 2008 the 
interviews were conducted by two senior PhD students. The "think-aloud" interviews largely consisted of open-ended questions that asked the students to explain their thinking processes in answering the various questions. Consistent with the approach followed by Steif and Hansen ${ }^{7}$, we deliberately did not interview students whose diagnostic test score was equal to or below the random-guessing score of 4.6. Similarly, since we wished to probe conceptual misunderstandings, we chose not to interview students with diagnostic test scores of 14 or higher. The interviews ${ }^{8}$ elucidated the following:

- $\quad$ Sufficient time was allowed for the test.

- The class did make a genuine effort in answering the test questions.

- The students were surprised at how much they had forgotten (although one could question whether they ever really understood the material). They reported that many of their peers also realized they had not done very well.

"...it was all stuff that we did actually learn in physics but after that long you sort of forget most of it."

"...from what I know most people just thought it was revision and we were also shocked at how much we forgot."

"Well it gave me quite a bit of a shock because... [in year-13] you just do it for the test and afterwards you kind of forget because you don't really use it or apply it in everyday life."

- $\quad$ Specific ad-hoc rules had often been taught in high schools (e.g. for motors and generators) and these were contrasted with the more-unified approach encountered in ELECTENG 101.

"Yes. There are two rules I know that."

"Yeah that one is only for movement and there's another for inducing which is kind of opposite to it. That's what I remember."

"I had the idea you make something move you use one rule and if you're using, you're moving something to make an electrical you just change it opposite it......I usually would just change it from my right hand to my left hand, just use it that way and it should be the opposite."

- $\quad$ Some students commented on a surface-learning approach at school versus a more conceptual approach at university. Not all were prepared to move away from a surfacelearning approach.

"I understood how to work out the equations and the calculations and so forth but I didn't get the concept"

"I pretty much just memorized"

"[At school] they try to cram these formulas in our head and make us memorize them without understanding the concept, so that's how we learnt. We just memorized formulas for the test. I hated it; I did not like school at all. Yeah there was no concept in our head, it was just formulas whereas in university we learn a lot more concepts."

As indicated above, interview transcriptions frequently highlighted a disjointed, rather than unified, student learning approach. In fact, one interviewee described the class in terms of conceptual learners (the minority) and rote learners (the majority). The recent national introduction of a module-based high-school qualification may have led teachers to adopt a more modular teaching style which, in turn, encouraged students to adopt a more fragmented approach to their learning. 


\section{A Closer Look at Some DC-Circuit Questions}

The 2008 diagnostic test results closely followed the 2007 results. In what follows, unless otherwise stated, the data are from the 2008 results.

In the question shown in Figure 2, only $61 \%$ of students selected the correct option (D), whereas Option (A) was selected by $29 \%$ of the students (37\% in 2007). When this question was answered by groups of high-school and university students in the United States ${ }^{2}$, only $40 \%$ selected the correct option while $44 \%$ selected Option (A). This indicates that a significant percentage of students have a conceptual model of electricity in which resistance $\mathrm{C}$ cannot affect bulb $\mathrm{A}$ because resistance $\mathrm{C}$ comes after bulb $\mathrm{A}$ in the circuit. Of course, they would have expected resistance $\mathrm{C}$ to affect bulb $\mathrm{B}$ because resistance $\mathrm{C}$ comes before bulb $\mathrm{B}$.

If you increase the resistance $C$, what happens to the brightness of bulbs $A$ and $B$ ?

(A) A stays the same, $B$ dims

(B) A dims, B stays the same

(C) $A$ and $B$ increase

(D) $A$ and $B$ decrease

(E) $A$ and $B$ remain the same

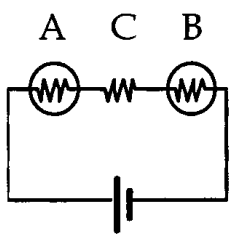

Figure 2. Diagnostic test, question 8.

Figure 3 shows another question for which student responses displayed a similar conceptual misunderstanding.

What happens to the brightness of bulbs A and B when the switch is closed?
(A) A stays the same, $B$ dims
(B) A brighter, B dims
(C) $A$ and $B$ increase
(D) $A$ and $B$ decrease
(E) $A$ and $B$ remain the same

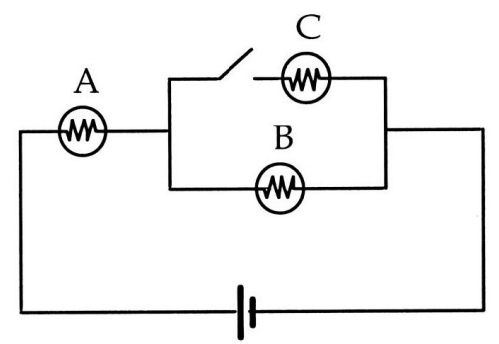

Figure 3. Diagnostic test, question 10.

Again, around one third of the class appeared to have a conceptual model in which 'later' components could not affect 'earlier' components. Option (A) was selected by $38 \%$ of the students (39\% in 2007). Presumably they thought that closing the switch could not affect bulb A since the switch came after bulb A in the circuit. These students would have expected the closing of the switch to affect bulb B, however: they would have reasoned that some of the current that previously passed through bulb B would now pass through bulb C, making bulb B dimmer. Just $26 \%$ of students selected the correct answer, option (B). The United States study referred to earlier ${ }^{2}$ returned similar figures: only $19 \%$ of students selected the correct option while $39 \%$ selected Option (A).

Clearly the misconception outlined above is well-established and widespread. Periago and Bohigas ${ }^{6}$ note that students should be instructed to "avoid sequential reasoning" in circuit theory: "The concept of an electric circuit as a whole would help us to combat another very common mistake, namely that any variation in a component in a circuit affects only components situated behind it". Engelhardt and Beichner ${ }^{2}$ also note students' propensity to 
use sequential reasoning. Such students "believe that current travels around a circuit and is influenced by each element as it is encountered, and a change made at a particular point does not affect the current until it reaches that point".

Significantly, the student response to the question shown in Figure 4 did not produce the same level of 'sequential-thinking' responses. Here 'only' 17\% of students (18\% in 2007) selected option (C), the option that sequential thinkers would select. The correct option of (B) was selected by $73 \%$ of the students. While the figure of $17 \%$ provides no comfort to instructors, it is certainly not as high as the figures of $29 \%$ and $38 \%$ returned by the two previous questions. This may be because some students viewed Circuit 2 as a new and distinct circuit, rather than as a change to Circuit 1 . In this case they would not apply sequential thinking.

How does the power delivered to resistor A change when resistor B is added as shown in circuits 1 and 2 respectively?
(A) Increases
(B) Decreases
(C) Stays the same

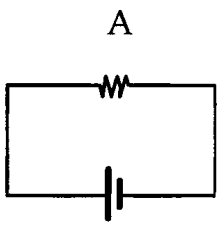

Circuit 1

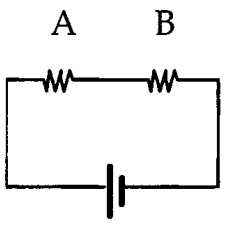

Circuit 2

Figure 4. Diagnostic test, question 1.

While a simple application of Ohm's law may have helped students to the correct answers for the questions illustrated above, some students arrived at quite nonsensical results through blindly relying on Ohm's law. For example, when asked the question shown in Figure 5, $41 \%$ answered option (A) (35\% in 2007). The correct answer, option (D), was chosen by $40 \%$ of the students.

If you double the current through a battery, is the potential difference across a battery doubled?

(A) Yes, because Ohm's law says $V=I R$.

(B) Yes, because as you increase the resistance, you increase the potential difference.

(C) No, because as you double the current, you reduce the potential difference by half.

(D) No, because the potential difference is a property of the battery.

(E) No, because the potential difference is a property of everything in the circuit.

Figure 5. Diagnostic test, question 7.

It is also likely that a blind reliance on Ohm's law led students to incorrectly analyse the question shown in Figure 6.

What is the potential difference between points A and B?
(A) $0 \mathrm{~V}$
(B) $3 \mathrm{~V}$
(C) $6 \mathrm{~V}$
(D) $12 \mathrm{~V}$

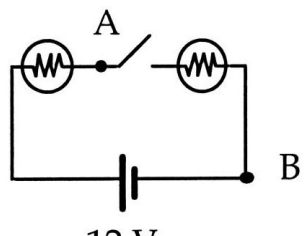

$12 \mathrm{~V}$

Figure 6. Diagnostic test, question 9.

Here $45 \%$ of students (44\% in 2007) chose option (A), perhaps reasoning that, as the current was zero, by Ohm's law the voltage had to be zero as well. Other researchers have also noted 
the propensity of students to apply to open circuits the notion that zero current implies zero potential difference ${ }^{9,10}$. Only $24 \%$ of students correctly answered (D). Remarkably, the United States study referred to earlier ${ }^{2}$ returned identical figures: $24 \%$ of students selected the correct option while 45\% selected Option (A). Disturbingly, when we put this question informally to a group of approximately 50 students who had been chosen on the basis of outstanding high-school academic achievement for direct entry to year-two engineering courses, only about $10 \%$ gave the correct answer (by a show of hands).

\section{A Closer Look at Some Electromagnetics Questions}

Figure 7 shows a diagnostic-test question used to determine whether students appreciate that a magnetic field exerts zero force on a stationary charge.

A positive charge $\mathrm{q}$ is held at rest in a uniform magnetic field, and then released.

You can ignore the effect of gravity on the charge.

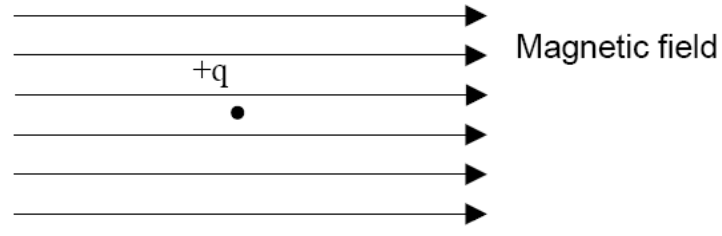

How does the charge move after it is released?

(A) The charge moves to the right with constant velocity

(B) The charge moves to the right with constant acceleration

(C) The charge moves in a circle with constant speed

(D) The charge moves in a circle with increasing speed

(E) The charge stays at rest

Figure 7. Diagnostic test, question 12.

The results for this question were of considerable concern, as they revealed significant misunderstandings of a fundamental and elementary concept. Only $25 \%$ of the students $(27 \%$ in 2007) gave option (E), the correct answer. By contrast 38\% thought the answer was option (B). Further investigation of the reasons for the above student responses is required. Even though great care was taken to stress the fact that the field was a magnetic field (this was mentioned both in the stem of the question and on the diagram), it may have been that many students still regarded the field as an electric field. Perhaps it would have made a difference if the field lines had been labelled with the symbol " $B$ " instead of the words "Magnetic field". Perhaps students might have handled the question better if it had been redrawn to show the electric charge between the poles of a magnet. There may be subtle issues with reading/literacy ability: perhaps students hardly read the question at all but simply looked at the diagram and thought it was an electric field. If students did realise that the field was a magnetic field, perhaps they have a conceptual model in which forces and field lines are always in the same direction, irrespective of the type of the field. Other researchers have noted the frequent occurrence of such a conceptual model ${ }^{4,11}$.

The above question was directly followed in the diagnostic test by the one shown in Figure 8 . In fact, it was considered that the statement offered as option (C) might have prompted some students to revisit the previous question and subsequently answer it correctly. 
How would you explain this?

(A) It experiences a steady force in the direction of the field lines

(B) It experiences a steady force opposite to the direction of the field lines

(C) There is no force on a stationary charge

(D) It experiences a steady force at right angles to its direction of motion

Figure 8. Diagnostic test, question 13.

The results for this question were also concerning, and consistent with those for question 12 . Once again significant misunderstandings were revealed, with only $22 \%$ of the students $(26 \%$ in 2007) selecting the correct answer, option (C). By contrast, $38 \%$ of the students selected option (A), a percentage which perfectly matches the percentage choosing option (B) in the previous question: at least it would appear that students realise that a constant force produces constant acceleration! This result raises the same issues as were raised in the previous question: did students take the field to be an electric field, or did they have a conceptual model in which forces and field lines are always in the same direction? It should be noted that $29 \%$ of students (28\% in 2007) selected option D, which might be explained by poor literacy skills (i.e. not reading correctly that the charge was initially at rest). Students may also have selected this distractor because it contained a familiar phrase that they had encountered previously.

The two questions discussed above probed student understanding of the force exerted by a magnetic field on a stationary charge. In the diagnostic test these questions were directly followed by two questions that probed student understanding of the force exerted by a magnetic field on a moving charge. The first of these questions is shown below in Figure 9.

The diagram below shows a positive charge $\mathrm{q}$ moving with constant speed $\mathrm{v}$ towards a region of uniform magnetic field.

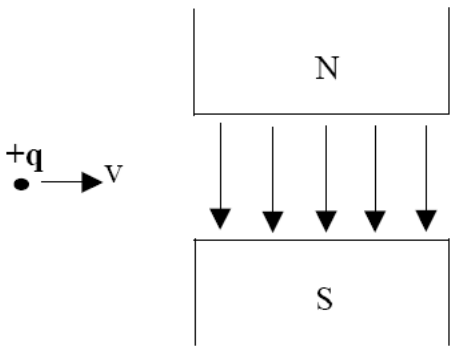

How does the charge move when it enters the field?

(A) It is deflected downwards $\downarrow$

(B) It continues moving at the same speed in the same direction

(C) It slows down and stops

(D) It is deflected into the paper

(E) It is deflected out of the paper

Figure 9. Diagnostic test, question 14.

While $38 \%$ of the students (39\% in 2007) selected the correct answer, option (D), a worrying $35 \%$ (34\% in 2007) selected option (A), indicating a "naive" approach where the force was simply considered to be in the direction of the field lines. (Similar proportions of the class displayed the same misunderstanding in questions 12 and 13.) We can take a little comfort from the fact that $22 \%$ selected option (E). While this is incorrect, it does at least demonstrate an understanding that the force must be at right angles to both the velocity and the magnetic 
field. Perhaps these students simply misused a hand-rule and so produced a force in the opposite direction to the correct direction.

In the diagnostic test, the question discussed above was directly followed by another that asked students to describe the path of the charge. This question is shown in Figure 10.

What is the path of the charge?

(A) A parabola (or part of a parabola)

(B) A circle (or part of a circle)

(C) A straight line

(D) Other

Figure 10. Diagnostic test, question 15, 2008.

The student responses for this question were puzzling. While in the previous question a total of $60 \%$ of the students had the charge deflected at right angles to the field lines, now only $26 \%$ were correct in having the charge following a circular path, option $(\mathrm{C})$. A remarkable $62 \%$ of students chose option (A), believing the path to be parabolic. In 2007 a slightly different set of options had been used for this question, as shown below in Figure 11.

What is the path of the charge?

(A) A parabola (i.e. the path of a projectile)

(B) A circle

(C) A straight line

(D) Other

Figure 11. Diagnostic test, question 15, 2007.

In 2007 , only $17 \%$ of students correctly perceived the path to be circular in nature, with $71 \%$ choosing the parabolic path option. These results suggest that the majority of students regarded the force as constant in direction, not appreciating that the force direction would change in step with the velocity direction. The "parabola" distractor was very powerful, perhaps as a result of the considerable time devoted to the study of projectile motion in highschool physics. The way the diagram was drawn was also reminiscent of the sorts of diagrams used in high-school lessons on projectile motion. It would be interesting to have students answer a question in which the magnetic field is normal to the plane of the page: perhaps such a question would produce a quite different result.

Questions very similar to 14 and 15 above were used with groups of high-school students of above-average ability in England and Turkey ${ }^{4}$. The results obtained paralleled those we obtained. For question 14, 25\% of the Turkish students and $43 \%$ of the English students answered correctly (c.f. 38\%). For question 15, the corresponding figures were $29 \%$ and $25 \%$ (c.f. 26\%). In all three countries, the "parabola" distractor was found to be very powerful.

Student understandings of the force exerted by a magnetic field on a charge were also probed by three further questions in the diagnostic test. These three questions $(18,19$ and 20) are reproduced below in Figure 12.

For question 18, a full $90 \%$ of the students (also $90 \%$ in 2007) correctly selected option (A), and therefore appeared to grasp the concept that a stationary electric charge in a magnetic field experiences zero force. However, question 12 tested this concept too, and only $25 \%$ of the students answered that question correctly. This difference needs further exploration. 
Clearly, students are approaching question 18 in some quite different fashion; possibly recalling a similar situation from memory. It may be fruitful to reword or redraw question 12 in some way; representing the magnetic field by crosses, for example, rather than by arrows.

The following information is for questions 18, 19 and 20:

Three identical metal bars are in the same uniform magnetic field. The direction of the field is into the paper $(\otimes)$.

Bar 1 is at rest, but Bars 2 and 3 are moving at a steady speed $\mathrm{v}$ in the directions shown.

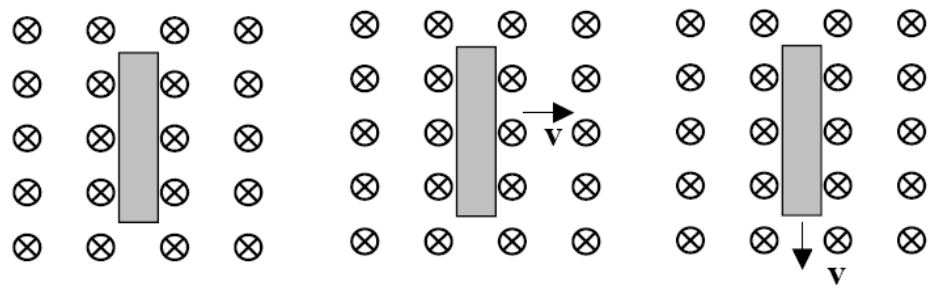

$\begin{array}{lll}\text { Bar } 1 & \text { Bar 2 } & \text { Bar } 3\end{array}$

Use the following options when answering the questions below:

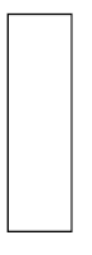

A

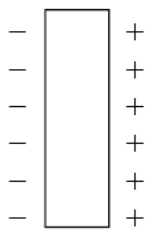

B

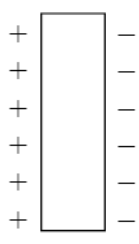

C

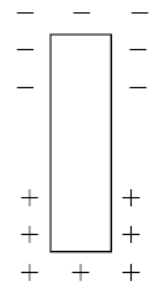

D

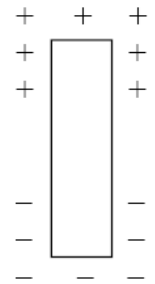

$\mathbf{E}$

\section{Question 18}

Which diagram shows the charge distribution in bar 1 ?

\section{Question 19}

Which diagram shows the charge distribution in bar 2 ?

Question 20

Which diagram shows the charge distribution in bar 3 ?

Figure 12. Diagnostic test, questions 18, 19 and 20.

Questions 19 and 20 essentially targeted the same concept, namely the polarity of the voltage induced in a conductor moving through a magnetic field, and returned very similar results. In question $19,30 \%$ of students correctly answered (E), with a further $22 \%$ of students getting the induced voltage direction reversed and choosing (D). In question 20, 29\% of students correctly answered (B), with a further $23 \%$ of students getting the induced voltage direction reversed and choosing $(\mathrm{C})$. These results are in keeping with those for question 14: about one third of students can correctly deduce the direction of the force on a moving charge and about one half of students know that the force is normal to both the field and the charge's velocity. Since the number of students getting the force direction the reverse of the correct direction is similar to the number of students getting the force direction correct, it is clear that there is considerable confusion when it comes to applying the ad-hoc hand rules that students have 
been taught in high-school physics. One interpretation of the above data is that only about $7 \%$ do know how to apply the rules correctly while a further $45 \%$ can apply both a right-hand rule and a left-hand rule but do not understand which rule to apply in a given situation. As part of the year-one course, students were taught a more unified vector-algebra treatment of electromagnetism which replaced the ad-hoc hand rules. Anecdotal feedback from student interviews indicated that most found the unified treatment more useful, but some students stuck with the hand rules regardless.

\section{Discussion}

Physics-education literature ${ }^{1-4,12}$ has consistently identified three major conceptual misunderstandings of dc circuit theory:

- The "sequential reasoning" fallacy

- Misapplication of Ohm's law

- The "current consumption" misconception

The first two of these were checked in our diagnostic test via choice of suitable distractors, while the third remains a work-in-progress to be checked by an amended version of the diagnostic test. Our results are aligned with those reported by others. A significant proportion of our students did exhibit the "sequential thinking" fallacy and did resort to blind (almost unthinking) misapplication of Ohm's law.

In the case of electromagnetic theory, reported conceptual difficulties ${ }^{4,11}$ include:

- Over-generalisation of correct or incorrect ideas from electrostatics

- Visualisation of forces and fields in three dimensions

- The "gut feeling" that force and field must be in the same direction

- Incorrect analogies between static charges and current carrying wires

- Difficulties in using the right-hand rule

- Difficulty with reasoning involving more than one step

- Confusion with change and rate of change of magnetic flux

While our diagnostic test doesn't include questions or distractors to test for all of these observations, we did observe problems with misuse of the right hand rule, confusion between electric and magnetic fields, assumptions that field lines always indicate the direction of the resulting force, and poor understanding of Faraday's law. In the case of Faraday's law, many students seem to confuse flux with induced voltage and many don't realise that a steady flux results in zero no induced voltage. These difficulties may be related to issues with reasoning involving more than one step.

We did also note some evidence of subtle issues with reading and literacy. After analysis of the 2007 diagnostic test results we slightly reworded some questions to reduce the effects of literacy issues as a possible confounder, before administering the 2008 diagnostic test.

When these results were shared with colleagues, even very experienced tertiary teachers were surprised at the extremely low (pre-tertiary) technical level at which significant misunderstandings were identified. If such misconceptions are not identified and corrected, it is very likely that the students will have significant difficulty picking up more advanced topics. Such difficulties may exacerbate retention problems, making it even more difficult for tertiary institutions to produce the increased number of graduates industry requires.

Consequently, an important related aspect of this research was to determine how effective incourse interventions were for correcting these misunderstandings. Rather than administer a 
corresponding post-test, selected diagnostic test questions were included in the final examination.

\section{The Diagnostic Test Revisited in the Final Examination}

The free-response question shown in Figure 13 was revisited in the 2007 final examination.

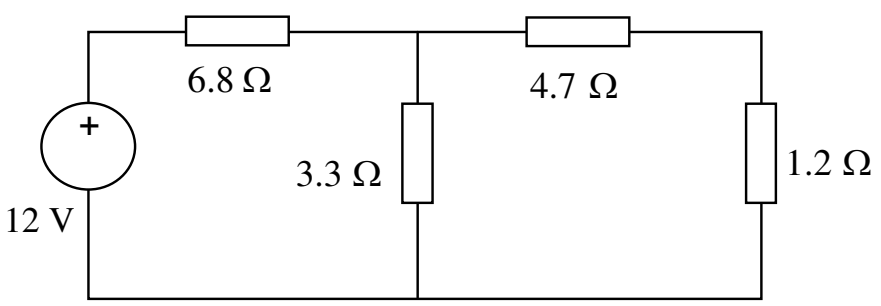

The diagram above shows four resistances connected to a voltage source. Find the current in the $1.2 \Omega$ resistance.

Figure 13. Diagnostic test, question 21

In the 2007 diagnostic test a mere $11 \%$ of the students answered the question correctly. Subsequently, tutors were asked to go over this question in the first tutorial. Tutors had not covered this question specifically in 2006. In addition, OASIS ${ }^{13}$, an on-line skills-based practice tool used in this course, contained this question and others like it for student practice. However, the set of OASIS questions offered for practice was the same in 2007 as it had been in 2006. In the final examination $78 \%$ of the students answered the corresponding question correctly.

The free-response question shown in Figure 14 was also revisited in the final examination.

$$
\text { Solve for } V: \quad \frac{V}{6}+\frac{V-3}{4}+\frac{V+5}{3}+2=0
$$

Figure 14. Diagnostic test, question 22

In the diagnostic test $60 \%$ of the students answered the question correctly. The corresponding final-examination question required students to analyse a circuit. The first step in the analysis was to derive an equation similar to that above. The second step was to solve the equation. $85 \%$ of the students could derive the equation and, of these, $94 \%$ correctly solved it. One lecture was spent on the circuit-analysis technique and, in the course of this lecture, three equations like the one given above were solved. Students were also given textbook, tutorial and OASIS problems that required them to solve such equations. None of this represented a change from previous practice, although the lecturer did remind the students of the diagnostic test question in the course of the lecture.

It did seem that the poor results in the diagnostic test served as a "wake-up call" for the students. In addition, modifications to course presentation to "plug the gaps" identified by the diagnostic test seem to have improved student understanding. This was confirmed by a significant improvement in examination performance from 2006 to 2007. Analysis of possible confounding issues, such as variations in cohort ability, alterations to marking schedules or 
adjustment of level of difficulty of questions, concluded ${ }^{14}$ that the potential confounding issues were insignificant.

\section{Conclusions}

A diagnostic test was administered in the second lecture of the ELECTENG 101 course with the aim of identifying the level of preparedness the student cohort brought to the year-one course. The test has now been administered to two successive cohorts comprising a total of over one thousand students. The results across the two years were very similar. More remarkably, similar questions used in diagnostic tests in other countries produced similar and, at times, identical statistics. The ELECTENG 101 diagnostic test acted as a "wake-up call" and led to behavioural changes on behalf of students and to some modification of course content. A question-by-question analysis has proven valuable, with the most significant feature to emerge from the analysis being the basic (pre-tertiary) level at which the misunderstandings occurred. The "think-aloud" interviews conducted with students were particularly helpful for identifying the "learning model" used by students. Analysis of student misconceptions of dc circuit theory and electromagnetic theory has identified a number of conceptual stumbling blocks for students. In particular, a significant number of students seem to follow a "sequential thinking" model in which variation in a component in a circuit affects only components situated behind it and many students blindly apply Ohm's law in inappropriate circumstances. Many students misuse the right hand rule, confuse electric and magnetic fields, assume field lines always indicate the direction of the resulting force, and exhibit a poor understanding of Faraday's law. Pleasingly, examination results show that such misunderstandings can be corrected by appropriate course interventions.

\section{Bibliography}

1. Maloney, D., et al., Surveying students' conceptual knowledge of electricity and magnetism. American Journal of Physics, Physics Educational Research Supplement, 2001. 69(7): p. S12 -S23.

2. Engelhardt, P.V. and R.J. Beichner, Students' understanding of direct current resistive electrical circuits. American Journal of Physics, 2004. 72(1): p. 98-115.

3. Ding, L., et al., Evaluating an electricity and magnetism assessment tool: Brief electricity and magnetism assessment. Physical Review Special Topics - Physics Education Research, 2006. 2(010105): p. 1-7.

4. Saglam, M. and R. Millar, Upper high school students' understanding of electromagnetism. International Journal of Science Education, 2006. 28(5): p. 543-566.

5. Holton, D., A. Verma, and G. Biswas. Assessing student difficulties in understanding the behaviour of $A C$ and DC circuits. in American Society for Engineering Education Annual Conference. 2008. Pittsburgh, PA.

6. Periago, M.C. and X. Bohigas, A study of second-year engineering students' alternative conceptions about electric potential, current intensity and Ohm's law European Journal of Engineering Education, 2005. 30(1): p. 71-80.

7. Steif, P.S. and M.A. Hansen, New practices for administering and analyzing the results of concept inventories. Journal of Engineering Education, 2007. 96(3): p. 205-212.

8. Smaill, C., E. Godfrey, and G. Rowe. The transition from final-year high-school physics and mathematics to first-year electrical engineering: a work in progress. in Eighteenth Annual Conference of the Australasian Association for Engineering Education. 2007. Melbourne, Australia.

9. $\quad$ Picciarelli, V., et al., A study of university students' understanding of simple electric circuits. I. Current in DC circuits. European Journal of Engineering Education, 1991. 16(1): p. 41-56.

10. Streveler, R.A., et al., Learning conceptual knowledge in the engineering sciences: Overview and future research directions. Journal of Engineering Education, 2008. 97(3): p. 279-294. 
11. Singh, C. Improving students' understanding of magnetism. in American Society for Engineering Education Annual Conference. 2008. Pittsburgh, PA.

12. McDermott, L.C. and P.S. Shaffer, Research as a guide for curriculum development: An example from introductory electricity. Part 1: Investigation of student understanding. American Journal of Physics, 1992. 62(1): p. 994-1003.

13. Smaill, C., The implementation and evaluation of OASIS: a Web-based learning and assessment tool for large classes. IEEE Transactions on Education, 2005. 48(4): p. 658-663.

14. Smaill, C., E. Godfrey, and G. Rowe. The transition from high-school physics to first-year electrical engineering: how well prepared are our students? in American Society for Engineering Education Annual Conference. 2008. Pittsburgh, PA. 